\title{
Slow Dentistry in Post Coronavirus 19 Era: Clinical Practice, Education and Well-being
}

\section{Nurcan Buduneli ${ }^{1}$, Fusun Ozer ${ }^{2}$, Saynur Vardar-Sengul ${ }^{3}$, Zeynep Burcin Gonen $^{4}$, Figen Cizmeci Senel ${ }^{5}$, Aylin Baysan ${ }^{6}$ and Sema S Hakki*}

${ }^{1}$ Department of Periodontology, Faculty of Dentistry, Ege University, Izmir, Turkey

${ }^{2}$ School of Dental Medicine, Division of Restorative, Dentistry, University of

Pennsylvania, USA

${ }^{3}$ Department of Periodontology, Nova Southeastern University, USA

${ }^{4}$ Oral and Maxillofacial Surgery, Genome and Stem Cell Center, Erciyes University,

Kayseri, Turkey

${ }^{5}$ Health Institutes of Turkey, Turkish Healthcare Quality and Accreditation Institute, Ankara, Turkey

${ }^{6}$ Barts and The London School of Medicine and Dentistry, Queen Mary University of

London, UK

${ }^{7}$ Department of Periodontology, Faculty of Dentistry, Selcuk University, Konya, Turkey

*Corresponding Author: Sema S Hakki, Department of Periodontology, Faculty of Dentistry, Selcuk University, Konya, Turkey.

DOI: $10.31080 /$ ASDS.2020.04.0985
Received: October 15, 2020

Published: November 18, 2020

(C) All rights are reserved by Sema $\mathbf{S}$ Hakki.,

et al.

\begin{abstract}
Objective: This review aims to critically evaluate the guidelines with respect to slow dentistry in clinical practice, education and well-being for staff and students in the post COVID-19 era.

Subject: According to the data released on 14 October, cases of COVID-19 have been spotted in 215 different countries, with $38,688,225$ total cases, 1,095,492 deaths and 29,035,243 recoveries. Since presence of asymptomatic infected cases is an unfortunate reality with COVID-19 and creating aerosols is a common occurrence in dentistry, the risk of cross-infection is high for patients and healthcare providers during dental procedures.

Results: Clinicians have treated emergency cases only to prevent possible spread of the virus at the beginning of the pandemic and during the lockdowns in various countries. The Disease Control and Prevention Centers have announced strict infection control protocols. Aerosol creating procedures are conducted preferentially in separate clinics with additional control measures and with longer intervals between patients to ensure a slow and safe re-opening.

Conclusion: The critical training of dental teams including students regarding the infection control procedures is of utmost importance. The 'slow movement' is another solution to manage the post-COVID-19 to provide the optimum patient care and student learning experiences. In addition, well-being of dental teams and students are paramount during these unprecedented times.
\end{abstract}

Keywords: COVID-19; Dentistry; Aerosols; Prevention; Slow Dentistry

\section{Introduction}

A progressive pneumonia-like respiratory syndrome, caused by severe acute respiratory syndrome-coronavirus 2 (SARS- COV2) was first detected in December 2019 in Wuhan, China [1]. The
SARS-Cov-2 virus is a new member of the coronaviridae family, also named as 2019-n-CoV in the recent terminology [2]. It is an enveloped, single-stranded, zoonotic character RNA virus belonging to the genus Betacoronavirus of the Coronaviridae family, like the oth- 
er severe acute respiratory syndrome coronavirus (SARS-CoV) and the Middle East respiratory syndrome coronavirus (MERS-CoV). SARS-Cov-2 has distinct epidemiological and clinical pathophysiological features from the previous SARS-CoV and MERS- CoV [3].

SARS-Cov-2 determines COVID-19 (coronavirus disease 2019), which is a highly contagious infectious disease with the major symptoms of low-grade fever, cough, fatigue, and breathing difficulties from mild dyspnea to severe pneumonia [4]. It can be transmitted from human-to-human particularly with coughing and sneezing. It was recognized as a global pandemic by the World Health Organization (WHO) on 11 March 2020 [5].

The main way of infective contagion, namely horizontal route includes direct transmission such as droplets of cough and sneeze or indirect contact with mucous membranes in a dental setting [6]. In addition, COVID-19 was identified in saliva of infected patients [7]. A 14-day medical observation period from exposure is crucial according to the recent data, which reported the mean incubation period of the SARS-Cov-2 was 5.2 days and the doubling time was 7.4 days [3].

To date, due to the lack of a specific treatment modality, biosafety measures are necessary to reduce transmission. Therefore, during the unpredictable pandemic, healthcare workers should be kept safe against the infection. It is advisable to revise guidelines, personal protective equipment (PPE) and produce standard operating procedures (SOP) for each clinical procedure by all team members i.e. dentists, dental hygienists/therapists and dental assistants due to the close contact to aerosols and the high risk of the contagious nature of the virus.

\section{Guidelines and algorithms}

Along with published guidelines, the scope of urgent and emergency treatments was listed. Although there are differences between countries and organizations, the general approach was to continuously provide urgent and mandatory treatments for the management of severe pain, dental and maxillofacial trauma, medical consultations, and life-threatening swelling and infections.

Given that pandemic has been around for about six months, randomized controlled or long-term observational studies are lacking. Currently available data are either based on very brief observations or are descriptive. An important aspect of the guidelines is the awareness and readiness of providers to protect their patients as well as themselves. Dentists should be fully aware of 2019-nCoV spread methods, how to identify infected patients and most importantly, self-protection methods.

Before this outbreak, "Standard Precautions" were described by the Center for Disease Control and Prevention (CDC) in the United States (US) in 199622 and pertain to contact with blood, any type of body fluids, secretions and excretions (excluding sweat) regardless of whether they contain blood, non-intact skin and mucous membranes. A secondary level of precautions, "TransmissionBased Precautions", was designed to reduce the risk of transmission of pathogens through contact, droplets or airborne routes. Standard precautions were reported to be adequate in the prevention of transmission of infectious diseases from dental aerosols. Therefore, it is postulated that the practice of Standard precautions by dental practitioners provides adequate protection against COVID-19 transmission and obviates its spread in the dental setting. It is, however, important to note that all available guidelines have advised increased infection control measures in the treatment of COVID-19 patients [8-10].

Delaying elective dental procedures increases the risk of urgent and emergency needs. Elective does not mean optional, but instead defines procedures that do not require urgent intervention Restoration of anatomy and non-vital function may be elective, however pose serious risk with prolonged delay. As the pandemic continues to evolve, it is recognized that dental settings might also need to deliver non-emergency dental care. Dental settings should balance the need to provide necessary services while minimizing risk to patients and dental healthcare personnel [8].

The re-opening process was undertaken to ensure that the COVID-19 related measures and health care services continue, to keep hospitals safe for patients with and without COVID-19 diagnosis, to ensure the safety of healthcare professionals, and to ensure equal access to and safety for all patients. The re-opening guides aims to regulate the working order and health services of the staff as much as possible. Clinicians are advised to regularly consult their own country's Ministry of Health and dental association for current information for requirements specific to their jurisdictions, including recognizing the degree of community transmission and impact, and their region-specific recommendations [8].

Re-opening process: Decision on re-opening and tele-dentistry

Re-opening dental practices require a team approach and the date can be determined by looking at the local case counts and 
deaths, especially for populations at higher risk for severe illness not to overwhelm the healthcare system and increase the number of cases. CDC reported "Interim Infection Prevention and Control Guidance for Dental Settings During the COVID-19 Response" on May 19, 2020. Re-opening needs to be phased to minimize the risk, and risk stratification should be implemented. Higher risk procedures and patient groups need to be introduced gradually to dental practices for elective procedures.

To provide care in the safest way, tele-dentistry may be implemented to reduce the need for visits and decrease the physical contact and thereby the contamination risk. Upon re-opening, especially in open space clinical settings including students, consider starting with non-aerosol procedures to give enough time to all staff acclimating to the new infection prevention protocols.

\section{Triage and patient management}

Triage is a rather new term introduced to dentistry after the pandemic of SARS-CoV-2. The word comes from the French word trier that means "to sort". By definition dating back to World War I, it means grouping the patients basing on the severity of their injuries and the likelihood of their survival. Indeed, this is a term that appeals most to periods with huge numbers of cases such as a world war or pandemic. Nowadays with the overwhelming cases, triage has an irreplaceable place within the context of preventive measures to limit the contagion of SARS-CoV-2 in medical and dental facilities. Triage enables the healthcare providers to decide the order of treatment required and the major expected outcomes are patient safety, and cost-effective care.

Triage could be considered as the first step of preventive measures to limit contagion in dental clinics. Telephonic pretriage could be performed and this might help to schedule the appointments in the best way. At this stage, all patients should be advised not to have any accompanying person, wear a mask and have minimum and only obligatory belongings with them while coming to the dental clinic. Upon arrival, investigation of the patient's current health status as well as the presence of possible risk factors should be done.

It is advisable to ask patients whether they currently have or recently had fever or other prominent symptoms of COVID-19 (coughing, sneezing, fever and difficulty in respiration). In addition, recent travel to the highly epidemic areas is a vital information on the decision-making process. All dental clinics are currently advised to have contactless thermometers and record the forehead temperature at the start of each and every visit. Body temperature of the patient should be $\leq 37.5^{\circ} \mathrm{C}$. This information should be provided systematically with a questionnaire by obtaining patient's written consent. Nowadays, digital recordings are preferred in treatment rooms.

Patients with positive sign(s) and symptom(s) of COVID-19 should be informed and referred to an appropriate medical center for further evaluation (Peng., et al. 2020).

All dental treatment interventions need to be postponed in such patients for at least 14 days and only emergency dental care should be provided. The triage procedures are unable to guarantee to discriminate patients with/without SARS-CoV-2 infection, however, they are straightforward to perform with no additional cost or effort. The best policy is always to handle all patients as COVID-19 positive and take the necessary precautions for infection control.

\section{Procedures related to aerosol generation in dentistry}

The small particles created during dental procedures are typically known as either dust or water droplets and they are noticeable for dental practitioners and patients. These particles are defined as either splatter or aerosols. Micro-organisms emanating from the mouth and respiratory tracks of patients may also filter into the aerosols. Therefore, the transmission of microorganisms into aerosols is one of the major health risks for dental healthcare providers also creating risk for cross-infection [11].

Aerosols are characterized as small sized particles less than 50 $\mu \mathrm{m}$ in diameter. These particles can remain airborne (carried by air) for extended periods of time before they attach to nearby surfaces or enter the respiratory passages. In contrast, particles larger than $50 \mu \mathrm{m}$ in diameter are called splatter. Unlike aerosol, splatter remains airborne for only short periods of time due to their larger size [12].

Bioaerosols are defined as aerosols consisting of particles of any kind of organisms; bioaerosols are identified by environmental factors such as humidity, airflow and temperature. They typically contain bacterial and fungal strains of various kinds as well as viruses. Speaking, breathing, sneezing, and coughing frequently generate aerosols [13]. Generation of aerosols and splatter during most dental procedures is almost unavoidable. The study of the spread of aerosol was first conducted by Micik., et al. who showed 
that streptococcal aerosols generated by high-speed dental turbines remained airborne for more than 24 hours and confirmed their impact on the health of patients and healthcare providers [14].

Saliva, nasal and throat secretion, dental plaque, gingival crevicular fluid, blood, tooth tissues and materials are some of the aerosols generated during dental procedures [11,12]. The use of the ultrasonic scalar tip and the rotary bur on a high-speed hand piece are two particular sources of aerosol. Air-water aerosols generated during dental treatment procedures emanate from the mouth of the patient and blends into the surrounding air, thus impacting its composition. The configuration of aerosol changes from one patient to another depends on the time of the day, site and type of dental procedures applied in the oral cavity [13]. The main aerosol generating dental procedures include preparing cavities for plastic restorations and preparations for extra-coronal restorations with the high-speed handpieces, use of rotary instruments for root canal treatments, removal of old restorations, supra and subgingival debridement and polishing of teeth, placement of dental implants, and surgical removal of teeth.

The main entry ways during aerosol generating procedures are the mouth, nose, and eyes (Peng., et al. 2020). Clinicians are currently advised to minimize aerosol generation when treating patients and focus on concocting infection control techniques and negative pressure procedures that limit aerosol secretion [15].

It has been confirmed that the level of COVID-19 in saliva is relatively higher than that in the oropharynx during the early stage of COVID-19 [16]. Therefore, dental healthcare providers, given their prolonged proximity to aerosols, face an acutely high risk for transmission and infection of corona virus. With recognition of the unfortunate fact that there is currently lack of consensus for treatment or vaccination available for COVID-19.

\section{The use of personal protective equipment (PPE)}

Wearing PPE during dental procedures is essential for prevention of transmission of pathogens, as well as infected biological materials. Personal barriers mainly include full-length fluid-proof gowns, goggles, face masks, respiratory protective devices, full-face shields or visor and double gloving [17]. Therefore, it is imperative that the use of PPE is paired with other infection control practices, including effective handwashing and using alcohol-based hand sanitizers, as well as the consistent enforcement of infection prevention practices.

\section{N95/FFP respirators and extended use}

The infection control precautions require wearing mask or N95 respirators/Filtering Face Piece (FFP) beside the other PPE including gloves, aprons for each procedure involving direct contact with the patient body fluids [10]. N95 respirators/FFP are designed to reduce the entry of small airborne particles with tight sealing and their filtration requirements.

However, there is no consensus among the healthcare organizations of different countries on wearing the surgical masks or N95 respirators during non-aerosol generating procedures $[9,18]$. In case of COVID-19 (+) or suspected patients, N95/FFP respirators (e.g. FFP-2 or higher) are essential in dental settings during aerosol-generating procedures [17]. Respiratory protections are disposable and can be used continuously up to four hours [19].

Due to COVID-19 pandemic, the global health systems have faced shortage of N95 respiratory mask or its equivalent FFP2/ FFP3 and reuses have been considered [20]. Several countries allowed extended use and/or reuse of N95 masks and FFPs. According to CDC guidance published during COVID-19, there are extended use and specific decontamination methods such as vaporous hydrogen peroxide, ultraviolet germicidal irradiation, and moist heat, and limited reuse of $\mathrm{N95}$ respirators [8].

On the other hand, emergency use authorization (EUA) by the US Food and Drug Administration (FDA) allows the use of unapproved medical products but does not allow sharing or reusing N95 respirators, surgical masks [21].

\section{Disinfection protocols}

Careful disinfection of all surfaces is of utmost importance for infection control in a dental clinic. Chemical disinfectants are effective in interrupting the spread of viruses. However, their effectiveness is highly related with factors like organic loading, contact time of the virus and by biocidal activity and concentration of the chemical agent. A disinfectant may be recognized as effective in decontaminating environmental surfaces if it causes a three $\log _{10}$ reduction in virus titer [22]. Not only those surfaces on a dental chair but also frequently touched surfaces such as door handles, chairs, desks are strongly recommended to be cleaned with disinfectant solutions after each dental intervention. The SARS-CoV-2 virus is supposed to be able to remain on inanimate surfaces such as metal, plastic, or glass for up to 9 days. It is advised to wait for 15 min before cleaning the surfaces so that the aerosols in the air fall 
on the surfaces and then the room should be ventilated for about 15 min preferentially by opening the window for an efficient air change and the possibly contaminated surfaces should be cleaned thoroughly at the same time.

The best options for surface disinfection are 70\% isopropyl alcohol and 2.5\% sodium hypochlorite and these chemicals should preferentially be in contact with the surfaces for approximately five min [23]. The concentration of sodium hypochlorite can be even higher (up to $10 \%$ ) for surfaces that are visibly contaminated with blood and/or saliva and lower (1\%) for floor and walls. Stone models and impressions can be disinfected by alcohol-based disinfectants. Cleaning the surfaces with regular soap first can be suggested prior to using the chemical disinfectants (CDC). Disinfectant chemicals should be used according to the recommendations of the manufacturers with regard to the concentration, duration of application, etc. All personnel using disinfectants should wear appropriate PPE and after chemical application, adequate ventilation should be provided before use. Environmental Protection Agency (EPA) approved disinfectants should be preferred as much as possible.

The surfaces should be cleaned starting from the upper ones towards the lower ones and also always from the relatively clean surfaces towards the contaminated surfaces. Chlorine-based disinfectants have the advantages of being cost-effective, having broad spectrum and being easily obtained. On the other hand, they can be corrosive, irritant, can cause discoloration on surfaces, and can be inactivated by organic substances. Hypochlorous acid ( $\mathrm{HOCl}$ ) solution has a broad-spectrum antimicrobial activity and can be used by fogging. It is a weak acid with pH 5 - 6.5 and considered as the most effective chlorine solution. It is odorless, non-costic, non-corrosive, and leaves no remnant when evaporated. It kills microorganisms by oxidation and is considered as a safe chemical but is not stable and should be used in a short time following preparation. A concentration of $200 \mathrm{ppm}$ is usually recommended to decontaminate inert environmental surfaces in a 1-min contact time. The contact time should be longer with less concentrated solutions. $\mathrm{HOCl}$ solutions applied as either a liquid or a fog at 20 to $200 \mathrm{ppm}$ of available free chlorine was effective to decontaminate the inert surfaces tested with various viruses [24].

Chlorhexidine $0.2 \%$ and benzalkonic chloride $0.2 \%$ were found be rather ineffective against the SARS-CoV-2 [25]. In a systematic review, it was noted that exposure of human coronavirus to 1,000 ppm NaOCl for 1 min achieved > 3 LRV. However, this data is based on experiments on stainless steel surfaces and further research on other possible surfaces in dental practices is required [26].

The use of mouth rinse before dental procedures

In order to reduce the dangerous levels of viral load in the droplets and aerosols, the use of preprocedural oral rinses are recommended. Preprocedural mouth rinses are especially recommended in situations when dental rubber dam cannot be used. Chlorhexidine or essential oil-containing mouthwashes could serve as particularly effective antiseptics for free floating oral bacteria. However, they have no impact on bacteria contained in a biofilm such as dental plaque and are unlikely to affect viruses and bacteria settled in nasopharynx [27]. Preprocedural $30 \mathrm{sec}$ mouth rinses containing oxidative agents such as $1 \%$ hydrogen peroxide or $0.2 \%$ povidone is recommended to reduce the salivary load of oral microbes (Peng., et al. 2020).

\section{Dental rubber dam isolation}

Using rubber dam is particularly effective in reducing the generation of saliva and blood-contaminated aerosol or splatter in situations when high-speed handpieces and dental ultrasonic devices are used. Additionally, extra high-volume suction for aerosol and splatter emission can be used [17].

\section{High-speed hand pieces with anti-retraction}

Micro-organisms such as bacteria and viruses may contaminate the air and water coming from the dental unit and can therefore lead to cross-infection. The regular handpieces can draw out and eject the contaminated fluids during dental procedures. It is particularly recommended that dental handpieces specifically designed with anti-retractive valves or other similar anti-reflux capabilities be used to protect cross-infection [17]. Reducing the speed of the rotary handpiece can also decrease the emission of aerosols. Sterilization of handpieces after each use is essential in ensuring their internal and external sterility as well as preventing cross-infection and contamination of water lines.

\section{High volume evacuation (HVE)}

Application of HVE during dental procedures removes saliva and water easily and quickly eliminating environmental contamination with aerosols by removing a large volume of air (up to 100 cubic feet air per minute) [12]. HVE is also capable of significantly reducing aerosol and splatter contamination generated during power-driven scaling [27]. 
Reducing bacterial aerosols in the dental clinics

There are different ways of reducing bacterial aerosols in dental clinics such as, maintenance of air conditioning and air ventilation systems, air conditioning filters and UV light lamps [13]. UV lamp, with $254 \mathrm{~nm}$ wavelength, shows high fungicidal, viricidal and bactericidal effects through DNA destruction and protein denaturation [11]. Compared to a single chair clinic, large open-plan multi-chair clinic would produce higher levels of aerosol. In open floor plan clinics, keeping at least 6 feet distance between patient chairs and place cleansable separators between units has been recommended by allowing enough time between patients to clean the clinic and air i.e. 30 - 60 min. Well-designed air cleaning systems with High Efficiency Particulate Air (HEPA) Filters, which capture particles less than $0.3 \mathrm{~mm}$, including bacteria and viruses, are highly recommended for use in the dental clinics [28].

\section{Going slow: Dental procedures in a post COVID-19 world}

Slow dentistry has been introduced in 2016 [29], and this concept is becoming a new norm due to the challenges in clinical dentistry in the post COVID-19 era. The slow method aims to standardize the quality of patient care with strict disinfection measures and to work efficiently and effectively. In this respect, patient safety is also paramount of care with thorough risk assessments and tailored management strategies. Therefore, slow dentistry focuses on the oral healthcare from the perspective of prevention, and management by biomimetic approaches using minimally invasive procedures. There are three components in slow dentistry: fourhanded dentistry; patient safety and experiences; and implementation of minimally invasive strategies.

\section{Four-handed dentistry}

Four-handed dentistry implies that the dentist and assistant, working as a team, performing dental treatment in a manner that has been carefully and deliberately planned also significantly decreasing the time in the chair.

\section{Patient safety and experiences}

Definition of patient safety is "reducing the risk of adverse events related to exposure to dental care across a range of diagnoses or conditions" [30]. A recent literature survey has listed leadership, teamwork, the provision of evidence-based care, communication, learning, and patient-centered approach as important domains (subcultures)/core values of patient safety [31]. Raising the awareness of issues related to patient safety and COVID-19 for all clinicians is crucial and could be achieved through the standard operating procedures (SOPs) that aim to improve patient safety and quality of oral health care.

\section{Minimally Invasive (MI) strategies}

Early detection of dental diseases and managing them with a personalized MI approaches need to be considered due to the limited use of aerosol generating procedures in a post-COVID-19 practice. The ultimate aim is to control the vicious restorative cycle of a tooth life which could lead to a potential tooth loss and also to provide optimum quality of life with a dignified future for patients in all ages.

\section{Prevention methods}

In the post-COVID-19 era, preventive methods should clearly dominate the dental practice so that less and less people will require interventions. The approaches that combine fluoride with other protective agents such as triclosan, chlorhexidine, probiotics, and dental products either containing casein phosphopeptide (CPP)-amorphous calcium phosphate (ACP) formula or bioglass have been reported to enhance the efficacy of fluoride to modify biofilms, diminish the cariogenic bacterial challenge [32] and to promote remineralization by inhibiting the demineralization [33].

When prevention is not enough: Atraumatic restorative treatment (ART)

Atraumatic Restorative Treatment (ART) was initially developed in the 1980s, as a method of restoring teeth in disadvantaged areas for the management of cavitated dental carious lesions using hand instruments and glass ionomer materials [34]. Nowadays, ART is becoming popular in developed world with evidence related to fluoride release of glass ionomer materials, simplicity of ART approach, and reduced use of local anesthesia. ART could be considered to be the cornerstone of MI approaches for cavitated carious lesion management in combination with caries risk assessment, patient education, and preventative strategies. With respect to the effectiveness of this approach, the most recent meta-analysis of the ART survival both for single-surface and multiple-surface restorations based on 29 publications reported that for single-surface ART restorations in permanent teeth over 3 to 5 years, the mean survival rates were $85 \%$ and $80 \%$, respectively and $86 \%$ for multiplesurface ART restorations in permanent teeth over one year [35].

ART has also been used in institutionalized older populations for the management of root caries. It was reported that $81 \%$ of

Citation: Sema S Hakki., et al. "Slow Dentistry in Post Coronavirus 19 Era: Clinical Practice, Education and Well-being”. Acta Scientific Dental Sciences 4.12 (2020): 68-77. 
ART restorations survived or were successful when compared to the conventional technique using glass ionomer cement modified with light-curing composite resin for a period of 12 months [36]. ART might be an important approach that could lead to a significant improvement of oral health in all ages especially where aerosol generating procedures are discouraged.

Overall, slow dentistry reflects a cultural shift in the pace of life in patient care. The 'slow movement' is another solution to manage the post-COVID-19 to provide the optimum patient care and experiences.

Education: Training dental team in clinical practice and universities

All clinicians, staff and students should be trained according to their SOPs and role playing should be practiced before re-opening. Training requires a behavioral change and therefore, enough time should be given for adaptation especially in open space dental clinics with multiple providers such as dental schools. Training should include not only the infection prevention but also understanding the SARS-CoV2 virus and the contamination routes of the virus to control fear and panic due to lack of understanding.

At the beginning of this period the questions were:

- How would the patients be treated safely?

- How would the clinical teaching take place?

- How would the students' learning experiences be?

- How would the academic work be provided?

Fortunately, immediate solutions for academic teaching were designed and online education with contemporaneous feedback has been successfully performed following the lockdown in many countries. Throughout the course of the pandemic, large numbers of virtual congresses, webinars, and meetings were performed to provide continuing professional development for dental team members and students. However, quality assurance of online platforms has not been considered yet.

\section{COVID-19: Staff support and well-being}

In these unprecedented times, physical and mental wellbeing of healthcare professionals is of utmost importance, since the vagueness is likely to trigger more stress and anxiety. In addition, uncertainty over redeployment, training and employment insecurities, information bombardment on social media and/or webinars and guidelines may make healthcare professionals feel trapped.
The WHO and Public Health England acknowledge the importance of healthcare workers considering positive coping strategies and embedding regular breaks during the day when working home remotely (WHO, 2020) whilst Public Health England guidance also outlines safe working recommendations, advising staff to take regular breaks [37]. In the UK, the current level of mental health and emotional support available for National Health Service (NHS) staff, as they tackle the COVID-19 pandemic with long shifts, being away from their families and making decisions on the use of resources which would have life and death effect on patients with COVID-19. Therefore, the NHS has launched a 'mental health hotline' for its staff and there are also a number of free digital apps available to NHS workers until December 2020. Online feedback hub which is private and anonymous for the healthcare workers has also been set up, so that the needs and experiences of the NHS workforce could be listened at these unprecedented times. The aim of this hub is to ask participants to share how they are feeling, what more could be done to support them, and how the healthcare services could adjust its communications as part of the COVID-19 response [37].

Despite these support measures are considered to be important during the crisis period, longer-term impacts of this pandemic are unknown. In addition, with all non-urgent elective care have been suspended for some time, there are currently increased pressures on the workload for clinicians after opening the dental practices. Therefore, slow dentistry as described in previous sections aims to provide a physically and mentally healthy workforce for clinicians. Finally, it should be noted that staff welfare needs to be considered seriously since post COVID-19 era is going to have a social, economic and psychological impact on everyone in the world.

\section{Psychological approach of the COVID-19 pandemic in dentistry}

The psychological effects on the COVID-19 pandemic are driven by many factors, including uncertainty about the duration of the crisis, lack of proven therapies or a vaccine, and potential shortages of PPE and limited availability and reliability of testing. All of these concerns are amplified by the rapid availability of information and misinformation on the internet and media. Stress and anxiety are based on uncertainty and fear of not feeling under control of the situation.

Trust in leadership has utmost importance in crisis such as global pandemics. Conscious leadership focused on the highest good for all is paramount. Stress levels drop when people trust their leaders whom make decisions based on peoples' values and 
well-beings. It is so important for leaders to be in constant, clear and honest communication with their employees, to be in gratitude for their service, discussing their safety and providing mental health support. Trust, compassion and kindness in communication are essential.

Using technology for social interaction while physical distancing should be practiced creating psychological support for the team. Weekly meetings to socialize and check how everyone is doing are very important during these difficult times. Using positive and factual approach to the crisis instead of fear based negative approach together with mindfulness and meditative tools are highly recommended to increase focus on neutral and positive approach.

Studies showed that those who reported a strong social support network had a lower degree of stress and anxiety, and a higher level of self-efficacy [38]. A qualitative study of medical residents during the 2003 SARS outbreak in Toronto showed that anxieties around personal safety and risk of contagion to loved ones conflicted with their professional duty to care [39]. Clear and rapid hospital communication is helpful to address the reactions of health care workers based on uncertainty or fear. Leadership at the hospital level, infection prevention and control services, and other supervising bodies need to be transparent and flexible, acknowledge uncertainty and provide clear evidence-based plans that will bolster workers' trust, confidence, and self-efficacy. This includes direction about hospital processes and appropriate provision of supplies and equipment, particularly in the face of potential shortages [40].

Healthcare workers who are self-isolating or under quarantine report symptoms of post-traumatic stress disorder, depression, stigmatization and fear of financial loss. Failure to ensure appropriate support could result in underreporting of symptoms and increase the risk of in-hospital transmission from those who work while sick against advice.

High risk of infection due to aerosol generating procedures among dentists and dental assistants instills fear of spreading the virus to their patients and families. It should be noted that some clinicians were encouraged not come to work and would rather quit dental carrier completely. Many older clinicians chose to retire due to the fear of dying due to COVID-19.

\section{Student well-being}

Stress and anxiety among students should be also taken into consideration. The uncertainty of their future profession and inter- ruption in their education especially in clinical courses put them in worry and fear. As leaders in dental education, the academicians need to develop compensating strategies for their interruption in education by substituting their clinical experience with simulation models and creating discussion groups in online platforms on case-based scenarios. When the clinics are reopened, increasing the number of clinical hours to give students more time in clinics could be considered since the future is unknown with regards to the next wave of COVID-19.

Having a creative and open mind in the process is extremely important as this crisis forces us to accept the situation and move on to provide action plans to compensate for the loss of time and clinical experience. Having a neutral mind to see the situation as is with all the reality helps us to come up with more efficient and factual solutions.

Since clinicians are experiencing fear and anxiety that they may contract the virus and their families may be exposed to this continuous stress, they feel the conflict on the other hand that they will not be able to provide what their patients need and fulfill their mission as health care providers. In a recent study, dental staff, who had medical issues exhibited higher psychological distress requiring mental health support and this indicates that mental health education should be included in the core dental curriculum for future dentists [40].

To support healthcare workers including dentists, their staff and medical/dental students, experts need to intervene to help protect their physical and mental health. The need for preventive measures (stress reduction, mindfulness, and educational materials), in-the-moment measures (hotlines, crisis support), and treatment (telepsychiatry for therapy, and medication if needed) could be considered.

\section{Conclusion and Future Projections}

The most stressful and tragic aspect of the COVID-19 pandemic has been the total interruption of social networks and crippling uncertainty for patients, clinicians, students, and dental educators. The field is vastly different from other academic disciplines in the sense that it is based heavily on patient care, which requires comprehensive education.

Going forward, it should be noted that one could adapt to a "new normal" and improve clinical conditions to meet current needs. 
That being said, a pandemic-induced economic distress has affected all industries across the globe, including the dental profession especially with slowing down clinical daily activities. In addition, there is lack of evidence and consensus for the best practice during these unprecedented times.

As a conclusion, it is advisable to be patient, resilient and adaptable. As clinicians, educators and researches, best approach is to rely on professional judgments for staff and student wellbeing, and continuously update the dental teams according to the concrete evidence.

\section{Bibliography}

1. Zhu N., et al. "A novel coronavirus from patients with pneumonia in China, 2019". The New England Journal of Medicine 382 (2020): 727-733.

2. Lu R., et al. "Genomic characterisation and epidemiology of 2019 novel coronavirus: implications for virus origins and receptor binding". Lancet 395 (2020): 565-574.

3. Li Q., et al. "Early transmission dynamics in Wuhan, China, of novel coronavirus-infected pneumonia". The New England Journal of Medicine 382 (2020): 1199-1207.

4. Huang C., et al. "Clinical features of patients infected with 2019 novel coronavirus in Wuhan, China”. Lancet (2020): 497-506.

5. Mahase E. "China coronavirus: WHO declares international emergency as death toll exceeds 200". BMJ 368 (2020): m408.

6. Antonovics J., et al. "The evolution of transmission mode". Philosophical Transactions of the Royal Society B 372 (2017): 20160083.

7. Sabino-Silva R., et al. "Coronavirus COVID-19 impacts to dentistry and potential salivary diagnosis". Clinical Oral Investigations 24.4 (2020): 1619-1621.

8. Center for Disease Control and Prevention (CDC). Interim Infection Prevention and Control Guidance for Dental Settings During the COVID-19 Response (2020a).

9. European Centre for Disease Prevention and Control (ECDC). Personal protective equipment (PPE) needs in healthcare settings for the care of patients with suspected or confirmed novel coronavirus (2019-nCoV), February (2020).

10. World Health Organization (WHO). Advice on the use of masks in the context of COVID-19 (2020).
11. Szymanska J. "Dental Bioaerosols as an occupational hazard in dentist's workplace". Annals of Agricultural and Environmental Medicine 14 (2007): 203-207.

12. Harrel SK and Molinari J. "Aerosols and splatter in dentistry". JADA 135 (2004): 429-437.

13. Leggat PA and Kedjarune U. "Bacterial aerosols in dental clinic: a review". International Dental Journal 51 (2001): 39-44.

14. Micik RE., et al. "Studies on dental aerobiology. I. Bacterial aerosols generated during dental procedures". Journal of Dental Research 48 (1969): 49-56.

15. Odeh ND., et al. "COVID-19: present and future challenges for dental practice". International Journal of Environmental Research and Public Health 17.9 (2020): 3151-3161.

16. Yoon JG., et al. "Clinical significance of a high SARS-CoV-2 viral load in saliva". Journal of Korean Medical Science 35.20 (2020): 1-6.

17. Barabari $\mathrm{P}$ and Moharamzadeh K. "Novel Coronavirus (COVID-19) and dentistry-a comprehensive review of literature". Dentistry Journal 8 (2020): 53-71.

18. Center for Disease Control and Prevention (CDC). Decontamination and reuse of filtering facepiece respirators (2020b).

19. Kobayashi LM., et al. "Extended use or reuse of N95 respirators during COVID-19 pandemic: An overview of national regulatory authority recommendations". Infection Control and Hospital Epidemiology (2020): 1-3.

20. Feng S., et al. "Rational use of face masks in the COVID-19 pandemic". Lancet Respiratory Medicine (2020).

21. US Food and Drug Administration FDA (2020).

22. Lloyd-Evans N., et al. "Chemical disinfection of human rotavirus-contaminated inanimate surfaces". Journal of Hygiene 97 (1986): 163-173.

23. Peditto M., et al. "Dentistry during the COVID-19 Epidemic: An Italian Workflow for the Management of Dental Practice". International Journal of Environmental Research and Public Health 17.9 (2020): 3325.

24. Park GW., et al. "Evaluation of liquid- and fog-based application of sterilox hypochlorous acid solution for surface inactivation of human norovirus". Applied and Environment Microbiology 73.14 (2007): 4463-4468. 
25. Kampf G., et al. "Persistence of coronaviruses on inanimate surfaces and their inactivation with biocidal agents". Journal of Hospital Infection 104.3 (2020): 246-251.

26. Gallandat K., et al. "A systematic review of chlorine-based surface disinfection efficacy to inform recommendations for lowsource outbreak settings". American Journal of Infection Control (2020): S0196-6553 (20)30311-4.

27. Devker N., et al. "A study to evaluate and compare the efficacy of preprocedural mouth rinsing and high-volume evacuator attachment alone and in combination in reducing the amount of viable aerosols produced during ultrasonic scaling procedure". Journal of Contemporary Dental Practice 13.5 (2012): 681-689.

28. Hallier C., et al. "A pilot study of bioaerosol reduction using an air cleaning system during dental procedures". British Dental Journal 209.8 (2010): 1-4.

29. Roig P. "Slow dentistry". Quintessence International 4.2 (2016): 87.

30. Shojania KG., et al. Making health care safer: a critical analysis of patient safety practices Evidence Report/Technology Assessment No 43 (Prepared by the University of California at San Francisco-Stanford Evidence-based Practice Center under Contract No 290-97-0013) AHRQ Publication No. 01-E058 (2001).

31. Sammer CE., et al. "What is patient safety culture? A review of the literature". Journal of Nursing Scholar 42 (2010): 156-165.

32. Li J., et al. "Anti-Caries Effect of Arginine-Containing Formulations in vivo: A Systematic Review and Meta-Analysis". Caries Research 49.6 (2015): 606-617.

33. Sleibi A., et al. "A Comparative Evaluation of Ion Release Characteristics of Three Different Dental Varnishes containing Fluoride either with CPP-ACP or Bioactive glass". Dental Material 35.12 (2019): 1695-1705.

34. Frencken JE., et al. "Twenty-five-year atraumatic restorative treatment (ART) approach: a comprehensive overview". Clinical Oral Investigations 16.5 (2012): 1337-1346.

35. de Amorim R G., et al. "Survival of atraumatic restorative treatment (ART) sealants and restorations: a meta-analysis". Clinical Oral Investigations 16 (2012): 429-441.

36. Lo E C., et al. "ART and conventional root restorations in elders after 12 months". Journal of Dental Research 85 (2006): 929932.
37. NHS England. NHS launches mental health hotline for staff tackling COVID-19 (2020).

38. Xiao H., et al. "The effects of social support on sleep quality of medical staff treating patients with coronavirus disease 2019 (COVID-19) in January and February 2020 in China". Medical Science Monitor 26 (2020): e923549.

39. Rambaldini G., et al. "The impact of severe acute respiratory syndrome on medical house staff: a qualitative study". Journal of General Internal Medicine 20 (2005): 381-385.

40. Maayan S., et al. "Distress among Dentists and Dental Hygienists in Israel". International Journal of Environmental Research and Public Health 17 (2020): 2900.

\section{Assets from publication with us}

- Prompt Acknowledgement after receiving the article

- Thorough Double blinded peer review

- Rapid Publication

- Issue of Publication Certificate

- High visibility of your Published work

Website: www.actascientific.com/

Submit Article: www.actascientific.com/submission.php

Email us: editor@actascientific.com

Contact us: +919182824667

Citation: Sema S Hakki., et al. "Slow Dentistry in Post Coronavirus 19 Era: Clinical Practice, Education and Well-being”. Acta Scientific Dental Sciences 4.12 (2020): 68-77. 